\title{
Comprehensive genetic characteristics of dystrophinopathies in China
}

\author{
Peipei Ma', Shu Zhang ${ }^{1}$, Hao Zhang ${ }^{1}$, Siying Fang ${ }^{1}$, Yuru Dong ${ }^{2}$, Yan Zhang ${ }^{3}$, Weiwei Hao ${ }^{3}$, Shiwen $\mathrm{Wu}^{1 *}$ (D) \\ and Yuying Zhao ${ }^{*}$
}

\begin{abstract}
Background: Dystrophinopathies are a set of severe and incurable X-linked neuromuscular disorders caused by mutations in the dystrophin gene (DMD). These mutations form a complex spectrum. A national registration network is essential not only to provide more information about the prevalence and natural history of the disease, but also to collect genetic data for analyzing the mutational spectrum. This information is extremely beneficial for basic scientific research, genetic diagnosis, trial planning, clinical care, and gene therapy.

Methods: We collected data from 1400 patients (1042 patients with confirmed unrelated Duchenne muscular dystrophy [DMD] or Becker muscular dystrophy [BMD]) registered in the Chinese Genetic Disease Registry from March 2012 to August 2017 and analyzed the genetic mutational characteristics of these patients.
\end{abstract}

Results: Large deletions were the most frequent type of mutation (72.2\%), followed by nonsense mutations (11.9\%) , exon duplications (8.8\%), small deletions (3.0\%), splice-site mutations (2.1\%), small insertions (1.3\%), missense mutations (0.6\%), and a combination mutation of a deletion and a duplication (0.1\%). Exon 45-50 deletion was the most frequent deletion type, while exon 2 duplication was the most common duplication type. Two deletion hotspots were calculated-one located toward the central part (exon 45-52) of the gene and the other toward the 5 'end (exon 8-26). We found no significant difference between hereditary and de novo mutations on deletion hotspots. Nonsense mutations accounted for $62.9 \%$ of all small mutations (197 patients).

Conclusion: We built a comprehensive national dystrophinopathy mutation database in China, which is essential for basic and clinical research in this field. The mutational spectrum and characteristics of this DMD/BMD group were largely consistent with those in previous international DMD/BMD studies, with some differences. Based on our results, about 12\% of $\mathrm{DMD} / \mathrm{BMD}$ patients with nonsense mutations may benefit from stop codon read-through therapy. Additionally, the top three targets for exon-skipping therapy are exon 51 (141, 13.5\%), exon 53 (115, 11.0\%), and exon 45 (84, 8.0\%).

Keywords: Dystrophinopathies, Duchenne muscular dystrophy, Becker muscular dystrophy, Mutation spectrum

\section{Introduction}

$D M D$ is the largest gene described in human beings, spanning more than $2.5 \mathrm{Mb}$ of genomic sequence, and consisting of 79 exons. Mutations in DMD result in Duchenne muscular dystrophy (DMD) or Becker muscular dystrophy (BMD), collectively called dystrophinopathies. Mutations that disrupt the reading frame generally generate unstable

\footnotetext{
*Correspondence: wu_shiwen@yahoo.com; zyy72@126.com

'Department of Neurology, the General Hospital of Chinese People's Armed Police Force, Beijing, China

${ }^{4}$ Research Institute of Neuromuscular and Neurodegenerative Diseases and Department of Neurology, Qilu Hospital, Shandong University, Jinan,

Shandong, China

Full list of author information is available at the end of the article
}

RNA and lead to the production of nearly undetectable concentrations of truncated proteins, resulting in DMD. However, mutations always maintain the reading frame in BMD patients, resulting in truncated, but partly functional, dystrophin [1]. The reading frame rule applies to $90 \%$ of cases and is usually used both to confirm diagnosis of dystrophinopathies and distinguish DMD from BMD [2, 3].

The prevalence of dystrophinopathies is about one in 3600 to 6000 live male births [4]. DMD patients present with rapid deterioration of ambulation in early childhood, with boys usually losing the ability to walk before 12 years old. BMD patients show a milder course with patients preserving ambulation ability through 16 years of age. 
Clinicians make a diagnosis of Intermediate Muscular Dystrophy (IMD) for the intermediate phenotype.

Multiplex ligation-dependent probe amplification (MLPA) is performed in patients with related syndromes first since deletions and duplications are identified in the majority of patients through this method and the method is the most cost-effective approach to screen for these mutations. Patients who are MLPA-negative need further sequencing to detect small mutations [5].

Current care recommendations, such as glucocorticoids, cardiac protection, respiratory support, and rehabilitative functional training, can improve quality of life but cannot reverse the clinical course or prevent the inevitable outcome. Potential therapies focus on DNA/RNA-based approaches, such as viral vector-based gene therapy (DNA-based), gene-editing technology based on Clustered Regularly Interspaced Palindromic Repeats (DNA-based), stop codon read-through approach (RNA-based) and the exon-skipping approach (RNA-based). Ataluren (PTC-124) makes it possible to read through the premature stop codon and restore protein translation. Almost $83 \%$ of all DMD mutations may benefit from exon-skipping therapy [6].

The development of clinical trials in China for dystrophinopathies require more detailed information about mutation characteristics, natural history, and standards of clinical care, even though some hospital-based datasets, such as the Children's Hospital of Fudan University database for dystrophinopathy in east China [7] and a comprehensive database in south China [8], already exist. Here, we analyzed genetic data of 1042 DMD/BMD patients based on a national registry database called "Chinese Genetic Diseases Registry" [9].

\section{Methods}

\section{Patients and data collection}

We started the Chinese Genetic Disease Registry (www.dmd-registry.com) in 2012 and registered muscular diseases, including DMD, BMD, spinal muscular atrophy, and other neuromuscular disorders. More than 1400 DMD/BMD patients from all over China registered from inception through August 2017. Patients predominantly came from the eastern and central parts of China, probably due to the influence of geographic location, economic levels, and medical conditions (Fig. 1). Data collectors and analysts were hired to collect, collate, and upload data, follow-up by telephone, and perform other data collection and analytical tasks. Of all the DMD/BMD patients registered in the database, more than 500 patients took part in our multidisciplinary clinic. All data in the database will continue to be updated regularly at the patients' outpatient visits or via telephone follow-up every 6 months.

\section{Diagnosis of dystrophinopathies}

The diagnosis of DMD/BMD is confirmed by at least one of the following methods: (1) dystrophin protein deficiency demonstrated by muscle biopsy; (2) large deletion or

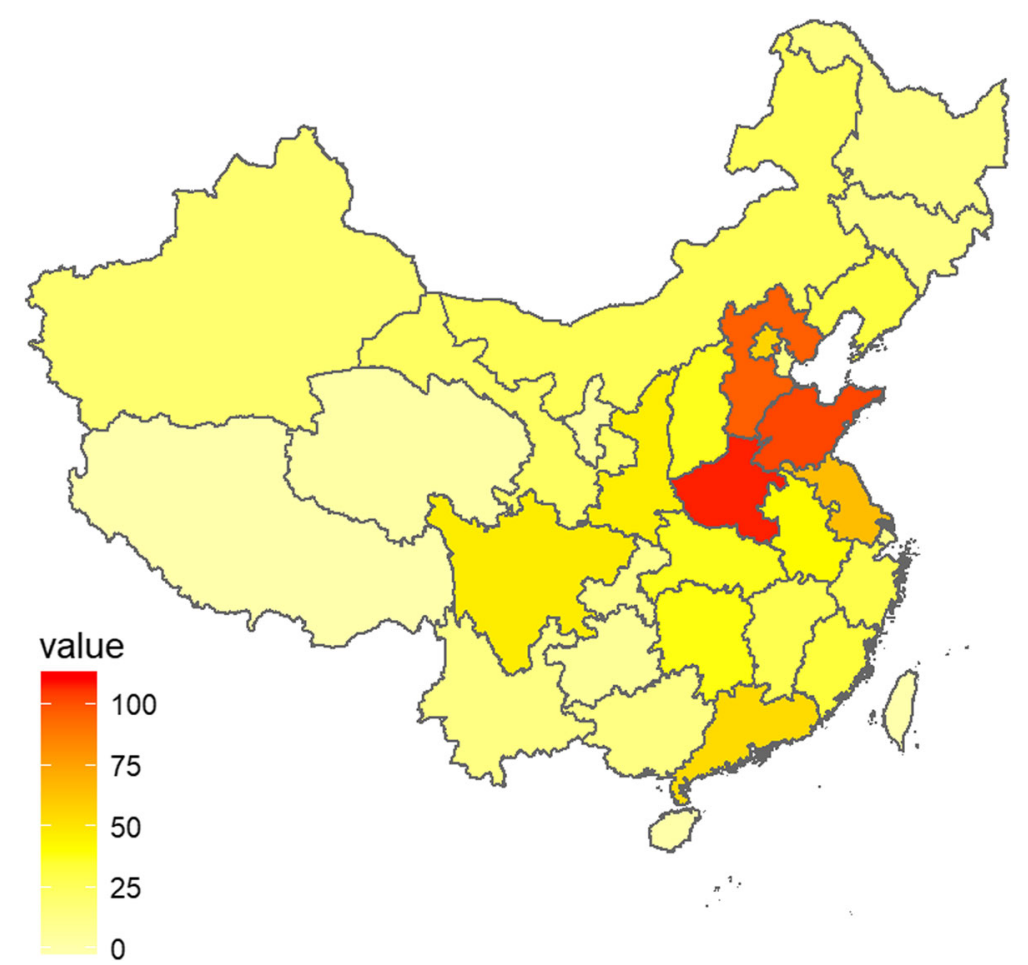

Fig. 1 The geographical distribution of patients in China 
duplication ( $\geq 1$ exon) detected by MLPA; (3) small mutations, including nonsense mutations, missense mutation, splice-site mutations, small insertions, or deletions demonstrated by complete dystrophin gene sequencing. Computer software called "DMD toolkit" was developed to visualize the structure of $D M D$ and to predict the functional changes of mutated dystrophin protein. In addition, the software helps improve the accuracy of clinical diagnosis [10].

\section{Results}

More than $1400 \mathrm{DMD} / \mathrm{BMD}$ patients registered in our database. Using genetic testing, we confirmed 1042 cases of unrelated DMD/BMD. Patients who underwent only hotspot sequencing using multiple polymerase chain reactions were excluded from this study.

Among the mutations from these confirmed patients, 845 were large mutations (81.1\%), of which 752 were large deletions ( $72.2 \%$ of all), 92 were large duplications $(8.8 \%$ of all), and 1 was a combination mutation of a deletion and a duplication ( $0.1 \%$ of all). Of the 197 small mutations (18.9\%), 124 were nonsense mutations (11.9\% of all), 22 were splice-site mutations (2.1\% of all), 31 were small deletions $(3.0 \%$ of all), 14 were small insertions (1.3\% of all), and six were missense mutations $(0.6 \%$ of all). Of the confirmed cases, 863 (82.8\% of all), 149 (14.3\% of all), and 30 (2.9\% of all) patients were diagnosed as DMD, $\mathrm{BMD}$, and IMD, respectively.

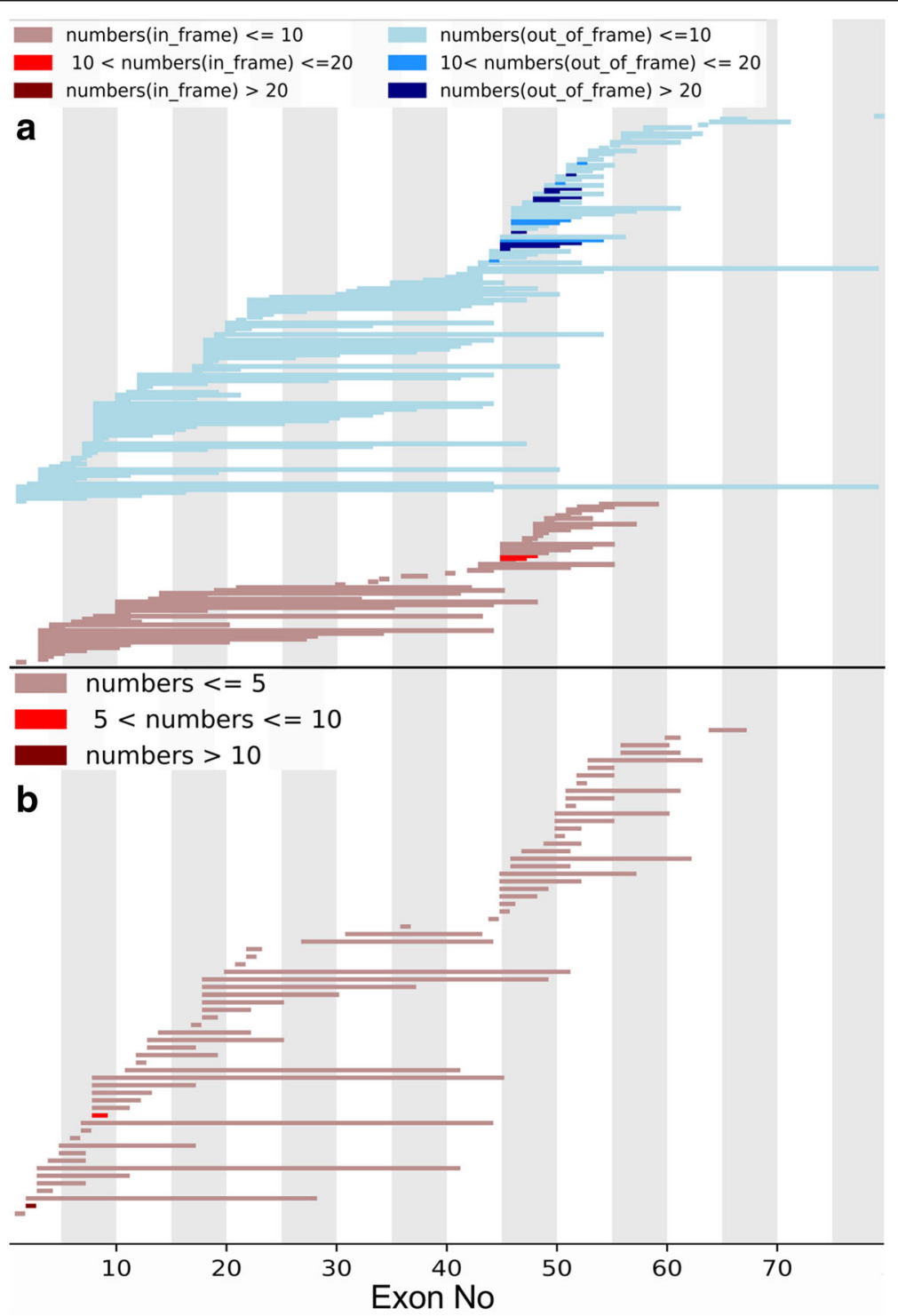

Fig. 2 Patterns of exon deletion and duplication in DMD in DMD/BMD patients. a Exon deletion: each bar represents a type of exon deletion. $\mathbf{b}$ Exon duplication: each bar represents a type of exon duplication 
Frequency and hotspot distribution analysis of large mutations (deletion and duplication of $\geq 1$ exon)

The deletion and duplication frequency is depicted in Fig. $2 \mathrm{a}$ and $\mathrm{b}$. The cumulative number of deletions and duplications is depicted in Fig. 3a and b. The exon 4550 deletion $(44 / 752,5.9 \%)$ was the most frequent deletion type, and the exon 2 duplication (13/92, 14.1\%) was the most common duplication type. Two deletion hotspots were observed: one located toward the central part of the gene and the other toward the $5^{\prime}$ end. The former was located in exons 45-52, which was the most common deletion region, containing up to $44.7 \%$ of all deletions. The latter hotspot included exons 8-26, taking up a smaller proportion (25.1\%) of all deletions. A duplication hotspot was present between exon 2 and exon 22, making up $41.8 \%$ of all duplications.

The frequency of deletions starting in the central hotspot (exons 45-52) comprised 69.4\% (522/752) of all deletions, while deletions starting in the proximal hotspot (exons 8-26) accounted for $12.5 \%$ (94/752) of all deletions. Large deletions affecting both hotspots were detected in seven patients $(0.9 \%)$. Whole-gene deletion (exon 1-79) occurred in one patient. We found 188 different deletion types in the database. Of those, 54 deletion types were detected starting in the central hotspot region, while 59 deletion types started in the proximal hotspot, indicating that the proximal hotspot had greater diversity.

The frequency of duplications starting in the hotspot (exon 2-22) was as high as 64.1\% (59/92). Duplications were more heterogeneous than deletions, with 66 types of duplication among 92 patients, 55 of which were reported only once in our database.

Two complex rearrangements were reported in our database: one patient held duplications in two different regions (exons 45-48 and exons 56-61), and the other patient harbored both a deletion and a duplication (exon 1 deletion and exon 2 duplication).

\section{Small mutations}

The 197 small mutations represented $18.9 \%$ of all mutations in our database and consisted of 124 nonsense
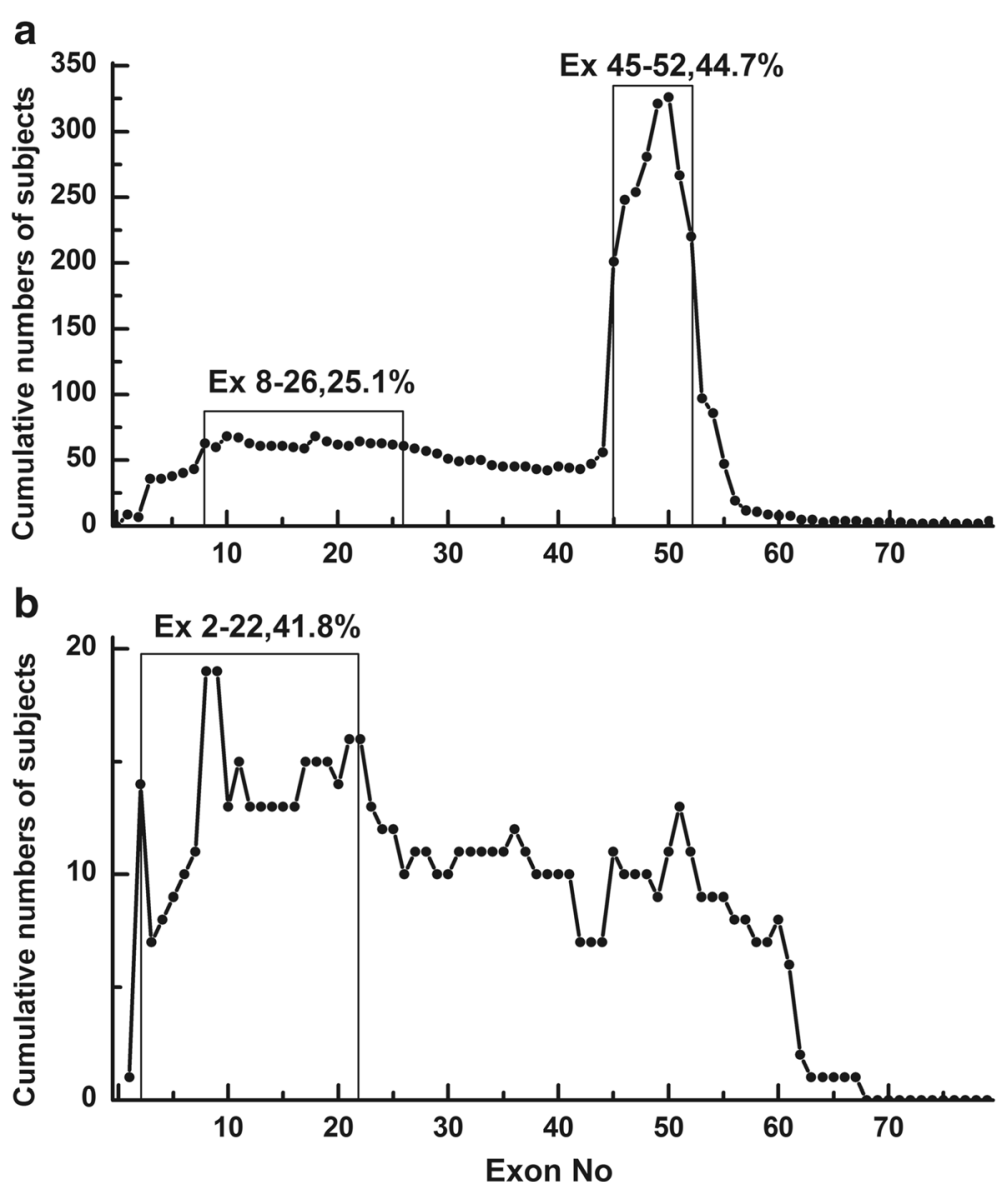

Fig. 3 Cumulative numbers of subjects with deletion or duplication. a Deletions: a hotspot is visible between exon 45 and exon 52. b Duplications: a hotspot is visible between exon 2 and exon 22 
mutations (62.9\%), 22 splice-site mutations (11.2\%), 31 small deletions (15.7\%), 14 small insertions (7.1\%), and six missense mutations (3.0\%) (Fig. 4a). Small mutations were varied and almost uniformly distributed throughout $D M D$ (Fig. 4b). Only 109 of the 197 small mutations were reported according to the Leiden Open Variation Database [11]. Details of the small mutations is contained in Additional file 1. Of the 124 patients with nonsense mutations, two were clinically diagnosed with BMD, 13 patients with IMD, and 109 patients with DMD. Although nonsense mutations were almost evenly distributed throughout $D M D$, some types of nonsense mutation appeared more frequently. c.433c $>\mathrm{T}, \mathrm{c} .583 \mathrm{C}>\mathrm{T}$, c. $8608 \mathrm{C}>\mathrm{T}$ and c. $2302 \mathrm{C}>\mathrm{T}$ were detected in $5,5,4$, and 3 patients, respectively.

\section{Carrier state analysis}

Genetic analysis was performed on mothers of 442 probands. Of those, 297 (67.2\%) possessed the same mutations as their children, while $145(32.8 \%$ )did not (Table 1). We analyzed deletion types and hotspot regions in the hereditary group and the de novo group, and the results are depicted in Figs. 5a, b, 6a, and b. We found no significant difference on deletion types and hotspot regions between the two groups, and the results were consistent with the deletion mutation distribution described above.

\section{Discussion}

Analysis of dystrophin mutations and their distribution could reveal potential targets for gene therapies. In this study, we analyzed the mutational characteristics of a group of Chinese DMD/BMD patients based on a large comprehensive database. The results of our analysis of the mutation spectrum or carrier state were consistent with the findings of other studies with slight differences [7, 8, 12-17].

\section{Mutation distribution}

Large mutations were the most prevalent mutation in many databases reported. Large and small mutations in

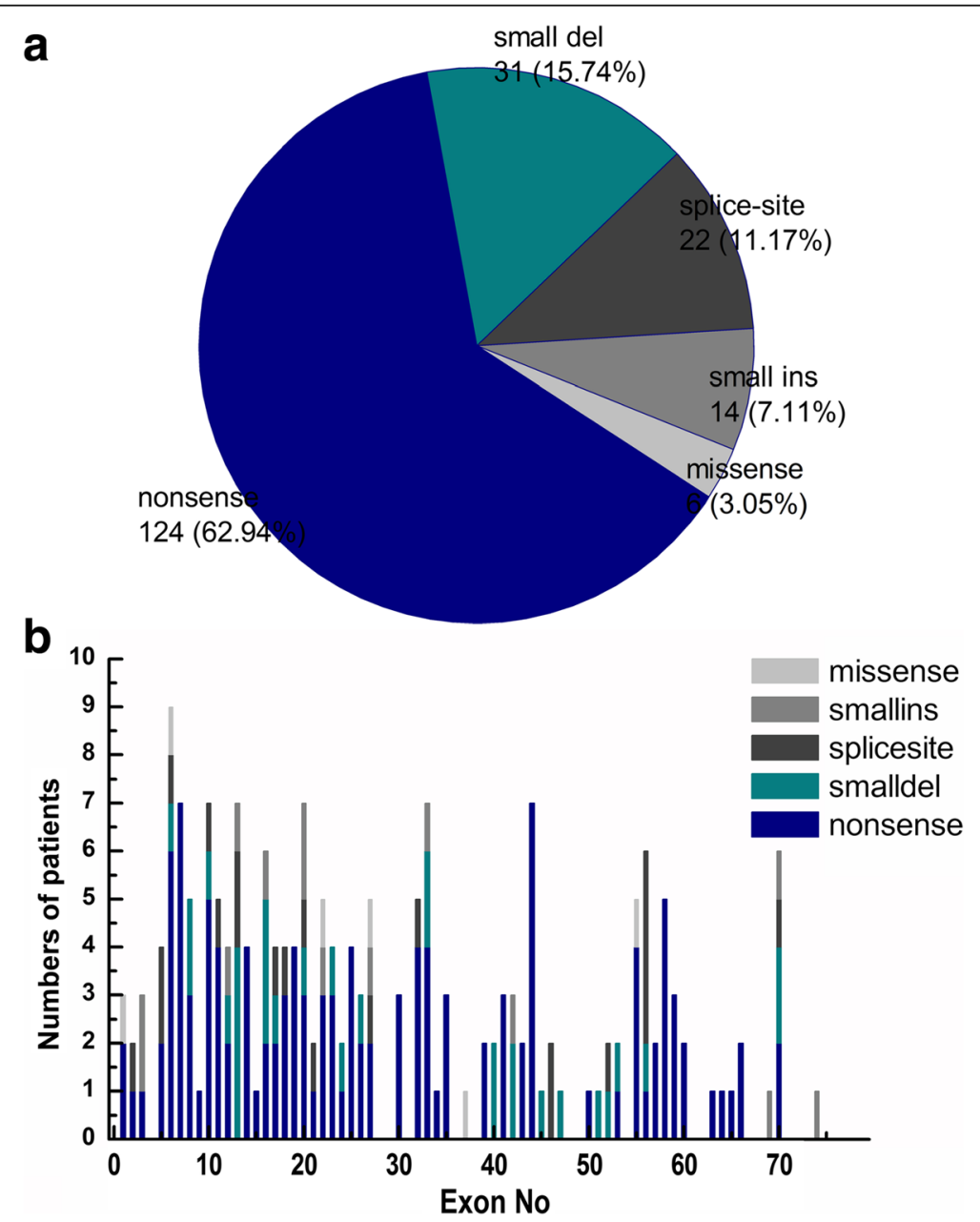

Fig. 4 a Small mutation spectrum in dystrophinopathy patients. b Distribution of small mutations in DMD 
Table 1 Carrier state analysis of mothers of 442 probands

\begin{tabular}{lllll}
\hline Mutation type & De novo & Hereditary & Total & Carrier rate \\
\hline deletion & 115 & 171 & 286 & 0.60 \\
duplication & 6 & 36 & 42 & 0.86 \\
nonsense & 16 & 57 & 73 & 0.78 \\
small del & 5 & 15 & 20 & 0.75 \\
small ins & 0 & 3 & 3 & 1.00 \\
splice site & 2 & 13 & 15 & 0.87 \\
missense & 1 & 2 & 3 & 0.67 \\
Total & 145 & 297 & 442 & 0.67 \\
\hline
\end{tabular}

this database were 81 and 19\%, respectively, which was similar to those in the Leiden database $(79 \% / 21 \%$ [12]), the TREAT-NMD DMD database $(80 \% / 20 \%$ [13]), and the French UMD database $(77.7 \% / 22.3 \%$ [14]). However, compared with our results, some of the previous studies from China demonstrated lower large mutation rates and higher small mutation rates $[8,15,18]$. For example, Dan-Ni et al. reported the rates of large and small mutations in 132 patients were 68.7\%/ $31.3 \%$ [8], respectively, which may be due to a smaller number of patients and the geographic concentration of registrants.

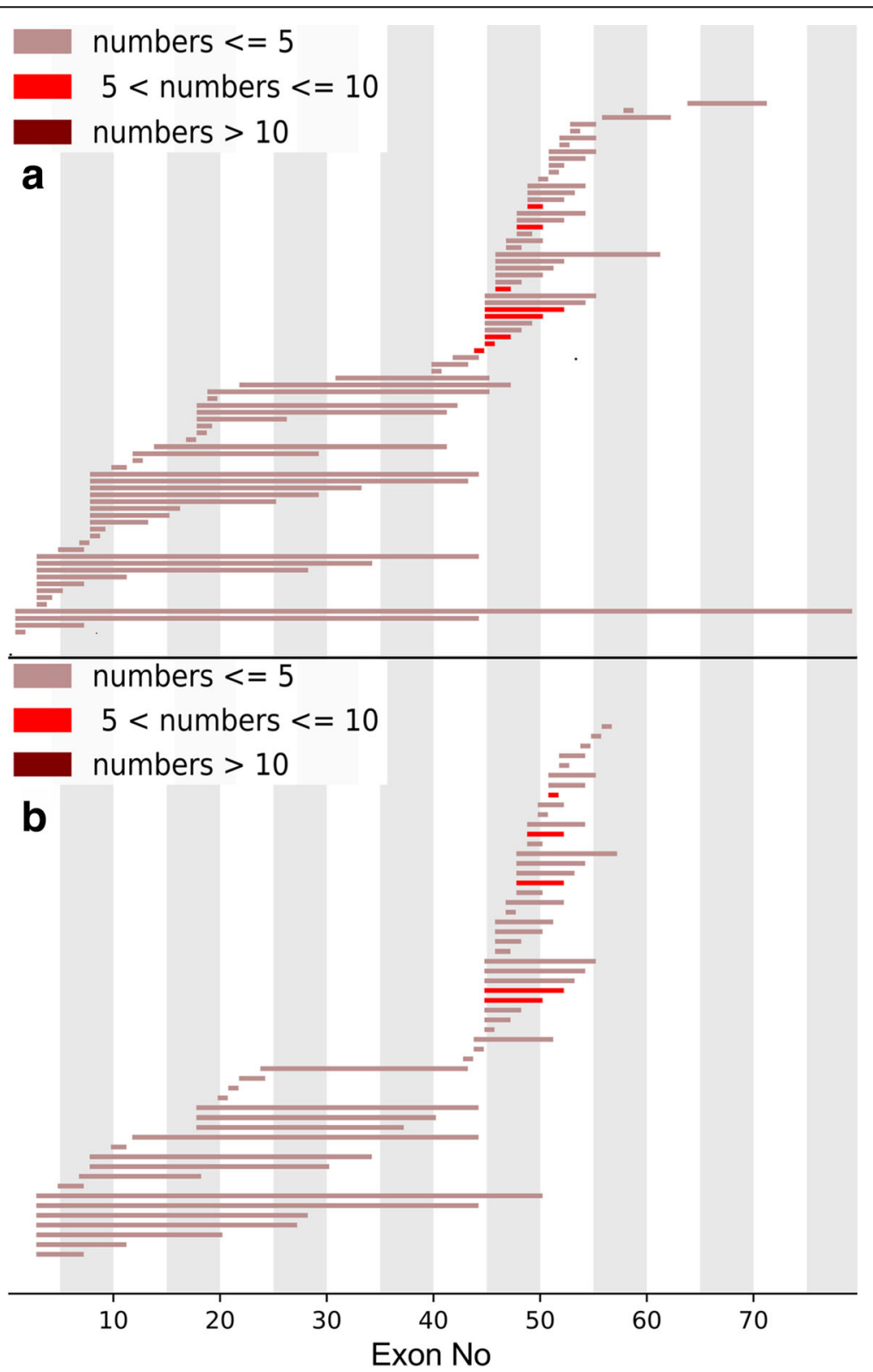

Fig. 5 Patterns of exon deletion in DMD in the hereditary group and de novo group. a Exon deletion in the hereditary group: each bar represents a type of exon deletion. $\mathbf{b}$ Exon deletion in the de novo group: each bar represents a type of exon deletion 

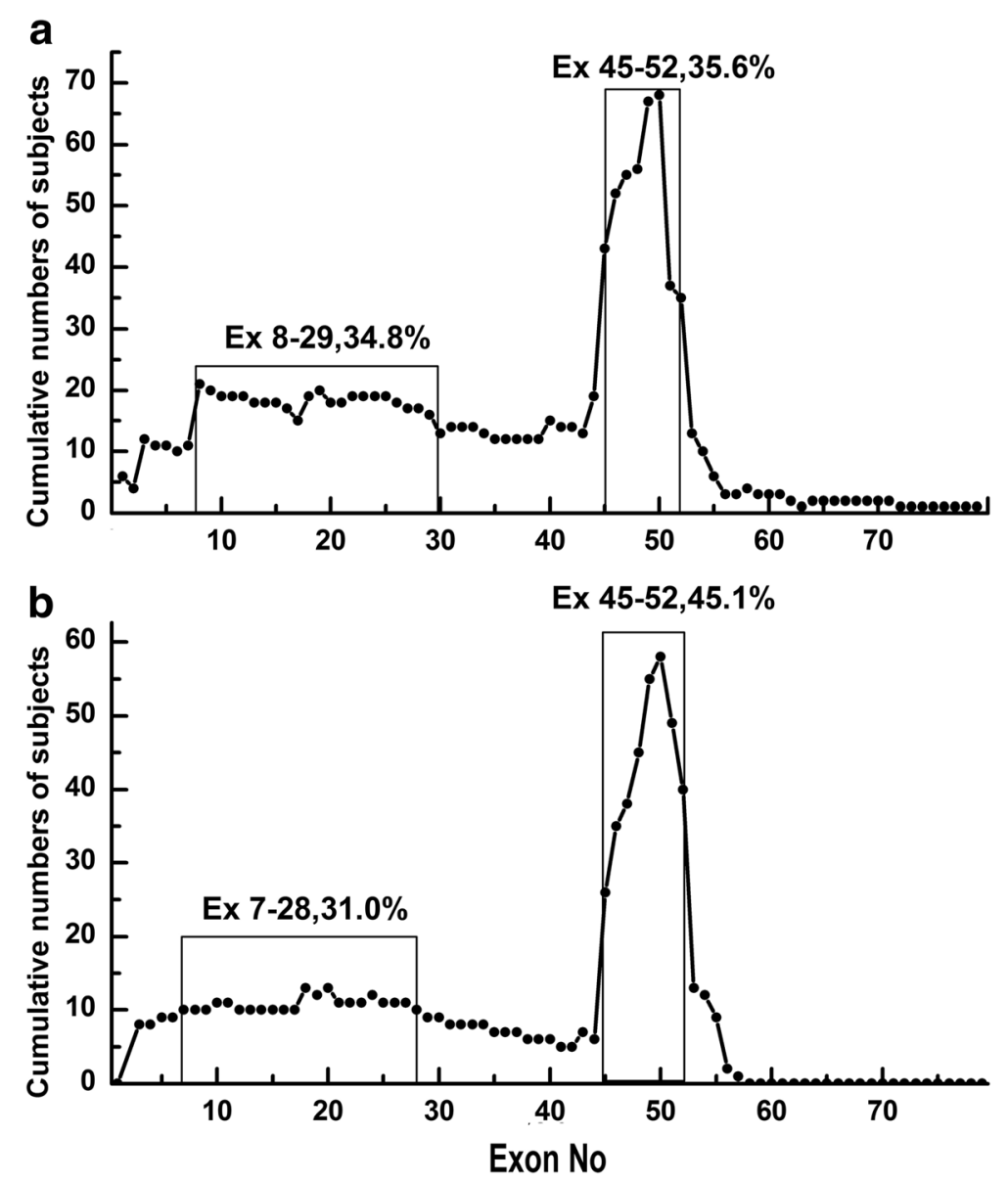

Fig. 6 Cumulative numbers of subjects with deletions in the hereditary group and the de novo group. a Deletions in the hereditary group. A hotspot is visible between exon 45 and exon 52. b Deletions in the de novo group. A hotspot is visible between exon 45 and exon 52

The most common mutations in $D M D$ were intragenic deletions, which accounted for $65 \%$ of the dystrophin mutations [19]. In our database, large deletions (72.2\%) and small deletions (3.0\%) accounted for $75.2 \%$ of all mutations. The most frequent deletion hotspot region in our database was between exon 45 and exon $52(44.7 \%)$, followed by the region between exon 8 and exon 26 (25.1\%). The results are consistent with those in other studies [7, 12, 15-17, 19]. Two hotspots were located in the central part of the gene and in the $5^{\prime}$ end, though deletions can happen almost anywhere in $D M D$ [19]. Some deletion types in hotspots were more frequently detected compared with others. For example, exon 4550 (4.2\% of all mutations) and exon 45 (3.5\% of all mutations) deletions were the most common deletion types in this study, while exon 45 constituted $4 \%$ of all mutations in the TREAT-NMD DMD database and $2 \%$ in the Leiden database. As we expected, exon 2-22 and exon 2 were duplication hotspots and the most common exon duplication pattern in this study and previous studies, respectively $[12-14,16]$.
Hotspot regions and most common mutation types (duplication, deletion) were similar worldwide, which suggests that the proportion of exon deletions and duplications in DMD/BMD had minimal variance based on ethnicity $[16,20]$ and that some dystrophin gene regions are vulnerable to rearrangement [21]. However, this issue remains a matter of controversy [22-24].

\section{Carrier state analysis}

Determination of carrier status is essential for both carrier screening and timely genetic counseling. Several DMD pedigrees contain more than one patient due to lack of knowledge about genetic counseling and prenatal diagnosis. Current genetic counseling practice is to attribute a carrier risk of two-thirds to the mother of a DMD patient [25]. In this study, we confirmed that the carrier rate for the mothers is $67 \%$. The carrier rate of deletion mutations was $60 \%$, while that of the other classes of mutations ranged from 75 to $86 \%$ (Table 1). Our data was also consistent with Haldane's theoretical model of de novo mutations in $\mathrm{X}$-linked diseases, as well as 
research in this field [26, 27], even though several smaller number studies showed that the carrier rate is much lower than the expected theoretical value [28-30].

Carrier state analysis revealed that exon 45-50 deletion was the most frequent deletions in both the carrier group $(9 / 171,5.3 \%)$ and the de novo group $(8 / 115,7.0 \%)$. Carrier state analysis also revealed a hotspot region located between exon 45 and exon 52, which was in approximately the same location as that of the whole study group. That suggests that no crucial difference exists between hereditary and de novo mutations.

\section{Potential therapies}

RNA-based therapies, such as stop codon read-through therapy and exon-skipping therapy, give hope to patients with nonsense mutations and large deletion mutations.

Table 2 The applicability of exon 51, exon 53, and exon 45 skipping for patients with deletion mutation

\begin{tabular}{lll}
\hline Skipped exon & Deleted exon & Number of patients \\
\hline 51 & 50 & 15 \\
52 & 20 \\
$17-50$ & 1 \\
$3-50$ & 1 \\
$30-50$ & 1 \\
$45-50$ & 44 \\
$47-50$ & 2 \\
$48-50$ & 26 \\
$49-50$ & 31
\end{tabular}

\begin{tabular}{ll}
53 & 20 \\
$43-52$ & 1 \\
$45-52$ & 30 \\
$47-52$ & 3 \\
$48-52$ & 30 \\
$49-52$ & 26 \\
$50-52$ & 5 \\
& total 115 (15.3\% of all) \\
44 & 18 \\
46 & 1 \\
$12-44$ & 2 \\
$18-44$ & 2 \\
$46-47$ & 26 \\
$46-48$ & 8 \\
$46-49$ & 8 \\
$46-51$ & 15 \\
$46-55$ & 2 \\
$46-57$ & 2 \\
& total 84 (11.2\% of all) \\
\hline &
\end{tabular}

Ataluren (Translarna $\left.{ }^{\mathrm{Tm}}\right)$ enables read-through of premature stop codons in mRNA to produce full-length and functional dystrophin protein and had been conditionally approved by the European Medicines Agency (EMA) for the treatment of DMD patients with nonsense mutation. Of the patients in this study, $11.9 \%$ may benefit from this therapy, $10 \%$ in the TREAT-NMD DMD Global database, 9.0\% in the French UMD database, 10.5\% in the Leiden database, and $13 \%$ in the Remudy database [12-14, 16]. All this data indicates that read-through therapy has significant potential in a wide range of clinical applications worldwide. Antisense oligonucleotide (AON)-mediated exon-skipping is another potential therapy for DMD patients that aims to produce partly functional proteins [31, 32]. Eteplirsen, used for exon 51 skipping, has been conditionally approved by the FDA and is now in clinical trials $[33,34]$. Meanwhile, clinical trials targeting exon 53 and exon 45 skipping were recently initiated [35]. However, exon 51, 53, and 45 skipping would cumulatively account for $32.5 \%$ of all patients in our database (Table 2). AONs targeting additional exons are still in the developmental phase and face many challenges [32, 36]. Thus, international registries providing detailed data is crucial to address these challenges.

\section{Conclusion}

The database for dystrophinopathies we created is a registry containing a wealth of information about patients with DMD/BMD, including mutation characteristics, family history, epidemiological data, natural history, motor function, cardiac function, respiratory function, management status, and survival time. Our analysis of the data collected thus far revealed a mutational distribution in this Chinese group largely consistent with that found in previous reports. This database provides a reference for basic research, facilitates clinical trials, and promotes the development of future gene therapy.

\section{Additional file}

Additional file 1: Clinical and genetic information of patients with small mutations. (PDF $93 \mathrm{~kb}$ )

\section{Abbreviations \\ BMD: Becker muscular dystrophy; CGDR: Chinese Genetic Disease Registry; CHFU: Children's Hospital of Fudan University; DMD: Duchenne muscular dystrophy; IMD: Intermediate muscular dystrophy; LOVD: Leiden Open Variation Database; LSDBs: Locus specific databases; MLPA: Multiplex Ligation-dependent Probe Amplification; Remudy: Registry of Muscular Dystrophy; TREAT-NMD: Translational Research in Europe-Assessment and Treatment of Neuromuscular Diseases \\ Acknowledgements \\ This study is supported by Department of Neurology, General Hospital of Chinese People's Armed Police Forces. We express our sincere gratitude for patients, families, physicians and the Beijing Zhiai Duchenne muscular dystrophy care center to support the national dystrophinopathies registry.}




\section{Funding}

This work was supported by Research and Development of Capital Clinical Characteristic Applications (No. Z151100004015025) and Beijing Municipal Science and Technology Commission. (No. Z151100003915126).

\section{Availability of data and materials}

The datasets generated and analysed during the current study are available in the Chinese Genetic Disease Registry repository (CGDR: www.dmd-registry.com). The detailed mutation information of the study are available on request.

\section{Authors' contributions}

PM participated in planning this study, analyzed and revised the data, and wrote the manuscript. SZ, HZ, SF, YZ and WH participated in data analysis, correction and interpretation. $Y Z$ and $W H$ also participated in generating part of the figs. SW and $Y Z$ supervised the planning of this study and guiding the writing of the manuscript. All authors read and approved of the final manuscript.

\section{Ethics approval and consent to participate}

The study was approved by research ethics committee and medical ethics committee, General Hospital of Chinese People's Armed Police Forces. All information used in the study were obtained from the General Hospital of Chinese People's Armed Police Forces, and were used for diagnostic purposes with written informed consent.

\section{Consent for publication}

Not applicable

\section{Competing interests}

The authors declare that they have no competing interests.

\section{Publisher's Note}

Springer Nature remains neutral with regard to jurisdictional claims in published maps and institutional affiliations.

\section{Author details}

'Department of Neurology, the General Hospital of Chinese People's Armed Police Force, Beijing, China. ${ }^{2}$ Department of Magnetic Resonance, the General Hospital of Chinese People's Armed Police Force, Beijing, China. ${ }^{3}$ Department of Precision Medicine Laboratory, the General Hospital of Chinese People's Armed Police Force, Beijing, China. ${ }^{4}$ Research Institute of Neuromuscular and Neurodegenerative Diseases and Department of Neurology, Qilu Hospital, Shandong University, Jinan, Shandong, China.

\section{Received: 18 April 2018 Accepted: 20 June 2018}

Published online: 04 July 2018

\section{References}

1. Monaco AP, Bertelson CJ, Liechti-Gallati S, Moser H, Kunkel LM. An explanation for the phenotypic differences between patients bearing partial deletions of the DMD. Genomics. 1988;2:90-5.

2. Koenig M, Beggs $A H$, Moyer $M$, Scherpf $S$, Heindrich $K$, Bettecken $T$, et al. The molecular basis for Duchenne versus Becker muscular dystrophy: correlation of severity with type of deletion. American journal of human genetics. 1989:45:498-506.

3. Nicholson LV, Johnson MA, Davies KE. Integrated dystrophin analysis using immunocytochemical,biochemical and genetic techniques. Basic and Applied Histochemistry. 1990;34:169-75.

4. Emery AE. Population frequencies of inherited neuromuscular disease-aworld survey. Neuromuscl Disord. 1991;1:19-29.

5. Aartsma-Rus A, Ginjaar IB, Bushby K. The importance of genetic diagnosis for Duchenne Muscular Dystrophy. J Med Genet. 2016;53:145-51.

6. Aartsma-Rus A, Fokkema I, Verschuuren J, Ginjaar I, van Deutekom J, van Ommen GJ, et al. Theoretic applicability of antisense-mediated exon skipping for Duchenne muscular dystrophy mutations. Hum Mutat. 2009:30:293-9.

7. Li X, Zhao L, Zhou S, Hu C, Shi Y, Shi W, et al. A comprehensive database of Duchenne and Becker muscular dystrophy patients ( $0-18$ years old) in East China. Orphanet J Rare Dis. 2015;10:5

8. Wang DN, Wang ZQ, Yan L, He J, Lin MT, Chen WJ, et al. Clinical and mutational characteristics of Duchenne muscular dystrophy patients based on a comprehensive database in South China. Neuromuscular Disorders. 2017:27:715-22

9. Chinese genetic disease registry. http://www.dmd-registry.com/. Accessed 31 Aug 2012.

10. Zhou J, Xin J, Niu Y, Wu S. DMDtoolkit: a tool for visualizing the mutated dystrophin protein and predicting the clinical severity in DMD. BMC bioinformatics. 2017;18:87.

11. Leiden Open Variation Database (LOVD). https://databases.lovd.nl/shared/ genes/DMD. Accessed 11 July 1997

12. Aartsma-Rus A, Van Deutekom JC, Fokkema IF, Van Ommen GJ, Den Dunnen JT. Entries in the Leiden Duchenne muscular dystrophy mutation database: an overview of mutation types and paradoxical cases that confirm the reading-frame rule. Muscle Nerve. 2006:34:135-44.

13. Bladen CL, Salgado D, Monges S, Foncuberta ME, Kekou K, Kosma K, et al. The TREAT-NMD DMD Global Database: analysis of more than 7000 Duchenne muscular dystrophy mutations. Hum Mutat. 2015;36:395-402.

14. Tuffery-Giraud S, Beroud C, Leturca F, Yaou RB, Hamroun D, MichelCalemard L, et al. Genotype-phenotype analysis in 2405 patients with a dystrophinopathy using the UMD-DMD database: a model of nationwide knowledgebase. Hum Mutat. 2009;30:934-45.

15. Wang X, Zheng W, Yan M, Huang S, Chen T-J, Zhong N. Similarity of DMD gene deletion and duplication in the Chinese patients compared to global populations. Behav Brain Funct. 2008;4:20.

16. Okubo M, Goto K, Komaki H, Nakamura H, Mori-Yoshimura M, Hayashi YK, et al. Comprehensive analysis for genetic diagnosis of Dystrophinopathies in Japan. Orphanet J Rare Dis. 2017;12:149.

17. Suh MR, Lee KA, Kim EY, Jung J, Choi WA, Kang SW. Multiplex ligationdependent probe amplification in X-linked recessive muscular dystrophy in Korean subjects. Yonsei Med J. 2017;58:613-8.

18. Chen C, Ma H, Zhang F, Chen L, Xing X, Wang S, et al. Screening of Duchenne muscular dystrophy (DMD) mutations and investigating its mutational mechanism in Chinese patients. PLoS ONE. 2014; https://doi.org/ 10.1371/journal.pone.0108038.

19. Muntoni F, Torelli S, Ferlini A. Dystrophin and mutations: one gene, severa proteins, multiple phenotypes. Lancet Neurol. 2003;2:731-40.

20. Banerjee $M$, Verma IC. Are there ethnic differences in deletions in the dystrophin gene? Am J Med Genet. 1997;68:152-7.

21. Mitsui J, Takahashi Y, Goto J, Tomiyama H, Ishikawa S, Yoshino H, et al. Mechanisms of Genomic Instabilities Underlying Two Common Fragile-SiteAssociated Loci, PARK2 and DMD, in Germ Cell and Cancer Cell Lines. Am J Hum Genet. 2010;87:75-89.

22. Baranov VS, Gorbunova VN, Malysheva OV, Artemyeva OV, Kascheeva TK Evgrafov OV, et al. Dystrophin gene analysis and prenatal diagnosis of Duchenne muscular dystrophy in Russia. Prenat Diagn. 1993:13:323-33.

23. Ballo R, Viljoen D, Beighton P. Duchenne and becker muscular dystrophy prevalence in South Africa and molecular findings in 128 persons affected. S Afri Med J. 1994:84:494-7.

24. Sinha S, Mishra S, Singh V, Mittal RD, Mittal B. High frequency of new mutations in North Indian Duchenne/Becker muscular dystrophy patients. Clin Genet. 1996;50:327-31.

25. Reid E. The calculation of genetic risks: worked examples in DNA diagnostics. 2rd ed. Johns Hopkins University Press;1994.

26. Haldane JB. The rate of spontaneous mutation of a human gene. J Genet 2004:83:235-44.

27. Emery AE. Population frequencies of inherited neuromuscular diseases - a world survey. Neuromuscul Disord. 1991;1:19-29.

28. Mukherjee M, Chaturvedi LS, Srivastava S, Mittal RD, Mittal B. De novo mutations in sporadic deletional Duchenne muscular dystrophy (DMD) cases. Exp Mol Med. 2003:35:113-7.

29. Alcantara MA, Villarreal MT, Del Castillo V, Gutierrez G, Saldana Y, Maulen I, et al. High frequency of de novo deletions in Mexican Duchenne and Becker muscular dystrophy patients. Implications for genetic counseling. Clin Genet. 1999;55:376-80

30. Bakker E, Veenema H, Den Dunnen JT, van Broeckhoven C, Grootscholten PM, Bonten EJ, et al. Germinal mosaicism increases the recurrence risk for 'new' Duchenne muscular dystrophy mutations. J Med Genet. 1989:26: 553-9.

31. Van Ommen GJ, Van Deutekom J, Aartsma-Rus A. The therapeutic potential of antisense-mediated exon skipping. Curr Opin Mol Ther. 2008;10:140-9.

32. Aartsma-Rus A, Straub V, Hemmings R, Haas M, Schlosser-Weber G, Stoyanova-Beninska V, et al. Development of Exon Skipping Therapies for 
Duchenne Muscular Dystrophy: A Critical Review and a Perspective on the Outstanding Issues. Nucleic Acid Ther. 2017;27:251-9.

33. Mendell JR, Goemans N, Lowes LP, Alfano LN, Berry K, Shao J, et al. Longitudinal effect of eteplirsen versus historical control on ambulation in Duchenne muscular dystrophy. Ann Neurol. 2016;79:257-71.

34. Aartsma-Rus A, Krieg AM. FDA Approves Eteplirsen for Duchenne Muscular Dystrophy: The Next Chapter in the Eteplirsen Saga. Nucleic Acid Ther. 2017; 27:1-3.

35. Fletcher S, Bellgard MI, Price L, Akkari AP, Wilton SD. Translational development of splice-modifying antisense oligomers. Expert Opin Biol Ther. 2017;17:15-30.

36. Aartsma-Rus A, Ferlini A, Goemans N, Pasmooij AM, Wells DJ, Bushby K, et al. Translational and regulatory challenges for exon skipping therapies. Hum Gene Ther. 2014;25:885-92.

- fast, convenient online submission

- thorough peer review by experienced researchers in your field

- rapid publication on acceptance

- support for research data, including large and complex data types

- gold Open Access which fosters wider collaboration and increased citations

- maximum visibility for your research: over $100 \mathrm{M}$ website views per year 